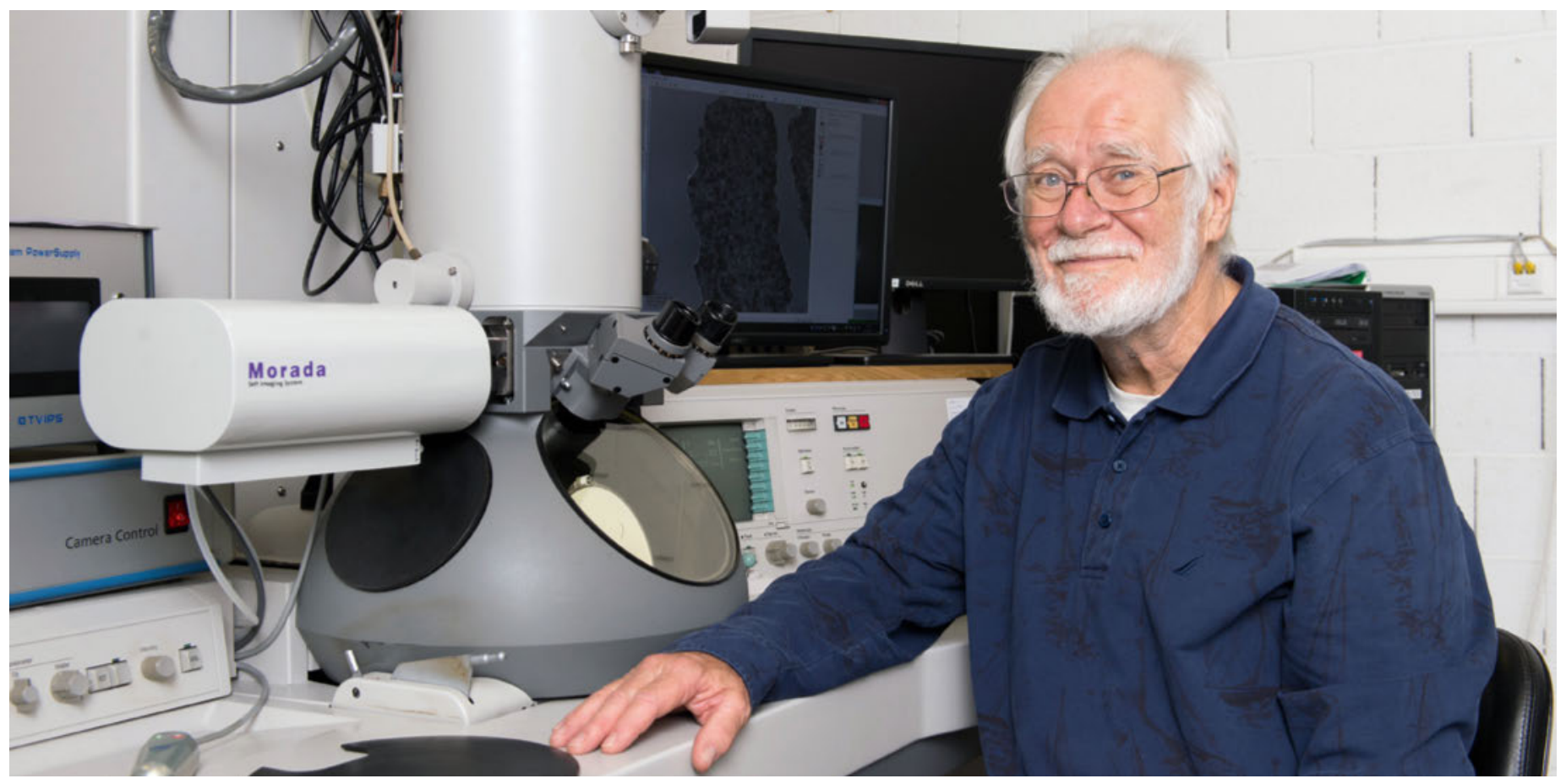

\title{
Garder son ancrage et manifester ses valeurs avec un Prix Nobel
}

\section{Jean Martin}

Dr méd., membre de la rédaction

"Qui, dans le grand public, connaissait Jacques Dubochet avant-hier?», écrivait le quotidien lausannois 24 heures au lendemain de l'attribution du Prix Nobel de chimie au professeur de l'Université de Lausanne. Quelques semaines plus tard on pourrait dire: «Qui, parmi ceux qui s'intéressent à la marche du monde et suivent l'actualité, n’a pas entendu parler de Jacques Dubochet?». Le Prix Nobel de Morges est un ami apprécié et, dans le tourbillon médiatico-sociéto-politique qui est son lot depuis le 4 octobre, il a bien voulu que nous nous assoyons pour échanger entretien cordial et stimulant.

Depuis des années, il était très connu dans le milieu de la recherche et son nom était mentionné comme nobélisable. Mais être un scientifique de haut niveau reconnu par ses pairs est une chose, devenir en quelques heures une personnalité mondialement visible en est une autre. "Avant le 4 octobre, je me construisais en paix sur le savoir, en moi et vis-à-vis des autres. Le 4 octobre, on me donne une voix incroyable et le pouvoir qui va avec.» Il a reçu quelque 1300 mails de félicitations, a été interviewé par les plus grands journaux et chaînes de télévision, a reçu 200 invitations à intervenir quelque part d'une manière ou de l'autre. Tout récemment, cela l'a gêné, dans une manifestation honorant un scientifique, on écartait ce dernier sans ménagement pour faire la place au nouveau Nobel. Son abord direct et sans façon, son intérêt pour des causes sociales, lui ont attiré de la sympathie, certaines remarques sur ce que sont la droite et la gauche en 
politique ont fait froncer des sourcils. Il relève avec gratitude la façon dont l'Université de Lausanne l'a soutenu dans la gestion de cette avalanche, mettant à sa disposition une personne compétente.

$\mathrm{Du}$ jour au lendemain, il est quelqu'un dont les avis sont recherchés sur tous les sujets. «Tout a changé pour

«Tu comprends, j'étais un scientifique... ce qui m'arrive n'a rien à voir avec ma vie antérieure»

moi, les choses ne seront plus jamais ce qu'elles étaient.» Il restera avec le poids - la notoriété, la gloire - de ce Nobel sur les épaules. Il s'agit pour lui de se familiariser avec les manières, les codes, mais aussi les surprises et les pièges, d'autres milieux que celui de la science: notamment des médias et de la politique (il a été actif de longue date au sein du législatif de sa ville de Morges mais les rencontres et invitations souvent prestigieuses qui lui arrivent sont d'un tout autre ordre). «D’abord ne pas céder à la 'peopolisation'. Ensuite ne pas dire de bêtises, ne pas m'exprimer, malgré les pressions, sur des thèmes que je ne connais pas suffisamment.» Conscient des défis, il entend s'entourer de conseillers critiques, en commençant au sein de sa famille et en élargissant le cercle.

Cela étant, ne pas croire que, avant, il était reclus dans son laboratoire. Dès son arrivée Lausanne en 1987, après des années de recherche ailleurs (Genève, le Biocentre de Bâle puis Heidelberg), il a animé des initiatives visant à l'ouverture. Ainsi, il a fait en sorte que la Faculté des sciences intègre le Séminaire d'éthique biomédicale, dont les partenaires étaient alors les seules Facultés de médecine, droit et théologie. Il a lancé un cours «Biologie et société», dont l'objectif explicite est de faire des étudiants d'aussi bons citoyens qu'ils sont biologistes. Il a œuvré une douzaine d'années dans un groupe cherchant à approfondir la réflexion et la pratique interdisciplinaires. Et tout au long il s'est engagé socialement, hors de l'université, en faveur des migrants notamment.

Même si aujourd'hui la relation avec les médias a un côté stressant, par l'urgence et la multiplicité des demandes, il a toujours été conscient de leur importance. Il s'en est préoccupé avec des collègues, invitant des journalistes pour mieux connaitre les intérêts, façons de faire, contraintes, des uns et des autres. Il suit de près les activités de l'Interface Sciences-Société établi à l'Université de Lausanne.

De multiples manières, Jacques D. s'intéresse donc aux enjeux sociétaux. Je lui parle d'humain augmenté, de enhancement. La première question, répond-il, est "Pour faire quoi, quel est le but?». Le simple allongement de la vie ne le convainc pas, et il est dubitatif quant aux compétences nouvelles que l'on pourrait vouloir inclure en nous. Rester réservé à l'endroit de "avancer pour avancer" sans qu'on pose clairement la question de la destination (me revient le mot d'un conseiller fédéral, il y a trente ans, «Nous sommes dans un train qui va très vite mais nous ne savons pas où»). Dubochet: «Dans le couple science-société, les scientifiques sont biaisés en faveur de la science. Il est dangereux de leur laisser la bride sur le cou.» A propos des poursuites obsédantes pour trouver toujours plus, mon interlocuteur exprime ainsi un mot de prudence «Il faut freiner»- ou, en tout cas, il faut consacrer assez de temps à réfléchir - dans une certaine «lenteur» - et définir dans quel sens on le fait, et dans quel cadre. M'est passé à l'esprit (J. M.) de poser la question de la considération qu'il convient de donner à l'utilité sociale potentielle de l'étude qu'on entreprend mais le temps n'a pas permis d'en discuter.

Nous parlons de Big Data, avec l'accroissement rapide des connaissances là où l'examen de grandes masses de données permet des avancées - avec des risques, notamment en termes de protection des données personnelles. «Il est important de se préoccuper du bon usage de Big Data", dit-il. Ici comme ailleurs se marque l'impératif d'une réflexion éthique, préalable dans toute la mesure du possible. Et de citer le titre du livre de 1995 de l'éthicien genevois Eric Fuchs, «Comment faire pour bien faire?», résumé pragmatique du propos de l'éthique. Jacques D. l'affirme fortement à plus d'une reprise, «le but qui me mène, c'est le bien commun».

L'échange sur l'éthique nous amène à l'état du monde, la croissance des inégalités au sein des pays et entre les pays (une situation incompréhensible, inadmissible, alors que nous disposons de tant de moyens), le peu de progrès dans une meilleure distribution des ressources et des chances, les conflits... Il insiste sur l'importance

\section{L'éthique, dimension qui doit être constamment considérée, aussi au niveau mondial}

d'aller vers des régulations fortes au niveau mondial, avec des instances qui aient des compétences de décision. L'OMS par exemple devrait avoir plus de moyens d'action en matière de santé. Il appelle de ses vœux un Parlement mondial. Quand je relève que j'ai le même souhait vif mais que la route semble encore bien longue, qu'on se demande si ce n'est pas illusoire, la réponse est «Illusoire peut-être, mais nécessaire!». Il rappelle cette phrase de Gramsci, reprise par d'autres, «Il faut allier le pessimisme de l'intelligence à l'optimisme de la volonté».

Nous revenons à ses préoccupations sociales. Depuis toujours, il s'est engagé pour des personnes en diffi- 
culté, laissées sur le bord du chemin. Posant des questions avec son franc parler, ne craignant pas de donner une impression de candeur. Cela apparait aussi quand il critique des positions élitistes et insiste pour que les académiques consacrent du temps et de l'énergie à faire œuvre de vulgarisation - parce qu'un tel échange maintiendra une confiance de la collectivité dans les chercheurs.

Question qui va de soi avec un biologiste, j'évoque l'évolution selon Darwin. "La théorie de Darwin, c'est du solide, qui a été confirmé par de nombreux éléments postérieurs à son auteur». Sans me faire l'avocat de l'«intelligent design", je mentionne Voltaire et son "L'univers m'embarrasse, et je ne puis songer que cette horloge existe et n'ait point d'horloger». Jacques D. fait alors référence aux durées d'évolution durant lesquelles ces choses se sont jouées (dizaines et centaines de millions d'années), dimension que nous ne sommes pas capables d'appréhender vraiment. L'évolution n'a pas de sens, le couple hasard/sélection a permis tout cela. Dans la foulée et à propos de dérèglement climatique (enjeu qui le préoccupe hautement, sur lequel il s'engage!), il souligne vivement que, là aussi, nous avons peine à l'appréhender mais dans un sens différent: nous peinons beaucoup à voir avec quelle incroyable rapidité le réchauffement intervient, une rapidité qui n'a rien à voir, n'en déplaise aux sceptiques, avec les changements qu'a connus la Terre au cours des périodes géologiques. Il veut avec d'autres que air, eau, lumière - et un climat vivable - restent des biens communs à tous.

«Nous peinons beaucoup à réaliser l'incroyable rapidité du changement climatique.»

Enfin, minute plus «soft», voire émue: revenant à son vécu depuis le 4 octobre, nous parlons de tous les messages reçus, de collègues, d'amis et de nombreux $M$. et Mme Tout le Monde. Des centaines de gens qui se disent heureux qu'il ait obtenu cette distinction suprême, qui manifestent à son égard tant de bienveillance, "presque de l'amour parfois, c'est très touchant». Je demande si ces correspondants disent aussi qu'ils comptent sur lui pour d'une façon ou de l'autre rendre notre monde meilleur... Peut-être bien.

\section{Crédit photo}

Félix Imhof @ UNIL

\section{Un Prix Nobel atypique, humaniste, engagé, pratique}

Le 4 octobre 2017, le Prix Nobel de chimie était attribué au biophysicien Jacques Dubochet (1942), de I'Université de Lausanne, ainsi qu'à deux collègues anglo-saxons, pour ses travaux dans le domaine de la cryomicroscopie électronique. Ses découvertes ont permis l'observation de matériel biologique à l'état natif. Cela implique de geler très rapidement, dans un bain d'éthane liquide, les échantillons à analyser afin de les figer dans leur état naturel, évitant une cristallisation en glace. Selon une de ses doctorantes: "L'apport scientifique est immense pour comprendre la structure moléculaire des cellules; cela a permis de voir très finement des virus en trois dimensions."

Devenu mondialement connu en une matinée, Jacques Dubochet a attiré l'attention par sa personnalité: d'un abord tout simple, avec un franc-parler plein d'humour («en quelque sorte, j'ai inventé l'eau froide», dit-il dans une conférence de presse impromptue). Un observateur: «Son CV hébergé sur le site de l'Université de Lausanne vaut son pesant d'éthane liquide». II dit être le "premier dyslexique officiel du canton de Vaud" - soutenu pour accéder à l'enseignement secondaire. Habitant de longue date de Morges, il est membre (PS) du législatif communal, et ses amis politiques relèvent qu'il a été leur homme-sandwich dans les rues de la ville lors de campagnes électorales... S'est attiré des froncements de sourcil en disant "L'altruisme est quelque chose de moral chez l'être humain, mais en biologie c'est une façon de fonctionner. Les fourmis ouvrières sont altruistes parce qu'elles travaillent pour la reine. L'altruisme est vital pour notre bien commun, ce n'est pas une valeur morale, c'est une notion d'intelligence: la gauche, c'est l'intelligence et la droite c'est l'égoïsme»! (1). On se réjouit de connaître son argument plus en détail.

jean.martin[at]saez.ch doise du $3^{e}$ âge - «ll m'a toujours frappé par sa préoccupation du plus grand nombre", dit la secrétaire générale. Ainsi que dans des mouvements à visée écologique, y compris l'association "Grands-parents pour le climat»; il était avec son épouse à la COP 21 de Paris à fin 2015. "Le changement climatique actuel est un évènement de taille comparable à la disparition des dinosaures il y a 65 millions d'années. Le gens ne réfléchissent pas de manière scientifique et n'arrivent pas à tirer les conséquences de la réalité.» [1]

Le premier sentiment qui lui est venu à l'esprit, au moment de la nouvelle arrivée de Stockholm, est la reconnaissance - à ses équipes, notamment celle du Laboratoire européen de biologie moléculaire de Heidelberg, où il a travaillé de 1978 à 1987. Et puis aussi le soulagement! "Cela fait des années que, dans les congrès, tout le monde me demande: ‘Alors, le Nobel, c'est pour quand?»" Commentaire sur l'évolution de la scène académique et scientifique: "Contrairement à ce qu'on exige des chercheurs aujourd'hui, je n'ai jamais eu besoin de courir après les publications. J'ai également eu le privilège, notamment grâce à l'Université de Lausanne, de ne pas avoir à passer mon temps à chercher des financements. J'ai eu la chance de profiter de conditions de travail exceptionnelles.» [2]

A noter enfin, Dubochet a introduit dans son enseignement universitaire, comme il l'a fait dans sa vie d'homme et de citoyen, des composantes de philosophie et d'éthique, parce que «avec la connaissance vient aussi une grande responsabilité».

\section{Références}

124 heures, Lausanne, 6 octobre 2017, p. 17-19

2 Le Temps Lausanne, 7 octobre 2017, p. 6-7, interview par Fabien Goubet. 